DOI: $10.1016 / j \cdot j$ spi.2013.01.007

\title{
Improved robust Bayes estimators of the error variance in linear models
}

\author{
Yuzo Maruyama ${ }^{*, \ddagger}$ and William, E. Strawderman ${ }^{\dagger, \S}$ \\ University of Tokyo $0^{\ddagger}$ and Rutgers University ${ }^{\S}$ \\ e-mail: maruyama@csis.u-tokyo.ac.jp; straw@stat.rutgers.edu
}

\begin{abstract}
We consider the problem of estimating the error variance in a general linear model when the error distribution is assumed to be spherically symmetric, but not necessary Gaussian. In particular we study the case of a scale mixture of Gaussians including the particularly important case of the multivariate- $t$ distribution. Under Stein's loss, we construct a class of estimators that improve on the usual best unbiased (and best equivariant) estimator. Our class has the interesting double robustness property of being simultaneously generalized Bayes (for the same generalized prior) and minimax over the entire class of scale mixture of Gaussian distributions.
\end{abstract}

AMS 2000 subject classifications: Primary 62C20, 62F15; secondary $62 \mathrm{~A} 15$.

Keywords and phrases: estimation of variance, harmonic prior, robustness.

\section{Introduction}

Suppose the linear regression model is used to relate $y$ to the $p$ predictors $x_{1}, \ldots, x_{p}$,

$$
\boldsymbol{y}=\alpha \mathbf{1}_{n}+\boldsymbol{X} \boldsymbol{\beta}+\sigma \boldsymbol{\epsilon}
$$

where $\alpha$ is an unknown intercept parameter, $\mathbf{1}_{n}$ is an $n \times 1$ vector of ones, $\boldsymbol{X}=\left(\boldsymbol{x}_{1}, \ldots, \boldsymbol{x}_{p}\right)$ is an $n \times p$ design matrix, and $\boldsymbol{\beta}$ is a $p \times 1$ vector of unknown regression coefficients. In the error term, $\sigma$ is an unknown scalar and $\boldsymbol{\epsilon}=\left(\epsilon_{1}, \ldots, \epsilon_{n}\right)^{\prime}$ has a spherically symmetric distribution,

$$
\boldsymbol{\epsilon} \sim f\left(\boldsymbol{\epsilon}^{\prime} \boldsymbol{\epsilon}\right)
$$

where $f(\cdot)$ is the probability density, $E[\boldsymbol{\epsilon}]=\mathbf{0}_{n}$, and $\operatorname{Var}[\boldsymbol{\epsilon}]=\boldsymbol{I}_{n}$. We assume that the columns of $\boldsymbol{X}$ have been centered so that $\boldsymbol{x}_{i}^{\prime} \mathbf{1}_{n}=0$ for $1 \leq i \leq p$. We also assume that $n>p+1$ and $\left\{\boldsymbol{x}_{1}, \ldots, \boldsymbol{x}_{p}\right\}$ are linearly independent, which implies that

$$
\operatorname{rank} \boldsymbol{X}=p .
$$

The class of error distributions we study includes the class of (spherical) multivariate$t$ distributions, probably the most important of the possible alternative error distributions. It is often felt in practice that the error distribution has heavier

\footnotetext{
*This work was partially supported by KAKENHI \#21740065 \& \#23740067.

${ }^{\dagger}$ This work was partially supported by a grant from the Simons Foundation (\#209035 to William Strawderman).
} 
tails than the normal and the class of multivariate- $t$ distributions is a flexible class that allows for this possibility. They are also contained in the class of scale mixture of normal distributions and thus, by De Finetti's Theorem, represent exchangeable distributions regardless of the sample size $n$.

In this paper we consider estimation of $\sigma^{2}=E\left[\left\{\sigma \epsilon_{i}\right\}^{2}\right]$, the variance of each component of error term, under Stein's loss (See James and Stein (1961)),

$$
L_{S}\left(\delta, \sigma^{2}\right)=\delta / \sigma^{2}-\log \left(\delta / \sigma^{2}\right)-1 .
$$

Hence the risk function $R\left(\left\{\alpha, \boldsymbol{\beta}, \sigma^{2}\right\}, \delta\right)$ is given by $E\left[L_{S}\left(\delta, \sigma^{2}\right)\right]$. The best equivariant estimator is the unbiased estimator given by

$$
\delta_{U}=\frac{\mathrm{RSS}}{n-p-1}
$$

where RSS is Residual Sum of Squares given by

$$
\mathrm{RSS}=\left\|\left(I-\boldsymbol{X}\left(\boldsymbol{X}^{\prime} \boldsymbol{X}\right)^{-1} \boldsymbol{X}^{\prime}\right)\left\{\boldsymbol{y}-\bar{y} \mathbf{1}_{n}\right\}\right\|^{2} .
$$

In the Gaussian case, the Stein effect in the variance estimation problem has been studied in many papers including Stein (1964); Strawderman (1974); Brewster and Zidek (1974); Maruyama and Strawderman (2006). Stein (1964) showed that

$$
\delta^{S T}=\min \left(\delta_{U}, \frac{\left\|\boldsymbol{y}-\bar{y} \mathbf{1}_{n}\right\|^{2}}{n-1}\right)
$$

dominates $\delta_{U}$. For smooth (generalized Bayes) estimators, Brewster and Zidek (1974) gave the improved estimator

$$
\delta^{B Z}=\phi^{B Z}\left(R^{2}\right) \delta_{U}
$$

where $\phi^{B Z}(\cdot)$ is a smooth increasing function given by

$$
\phi^{B Z}\left(R^{2}\right)=1-\frac{2\left(1-R^{2}\right)^{(n-p-1) / 2}}{n-1}\left\{\int_{0}^{1} t^{p / 2-1}\left(1-R^{2} t\right)^{(n-p-1) / 2} d t\right\}^{-1}
$$

and $R^{2}$ is the coefficient of determination given by

$$
R^{2}=\frac{\left\|\boldsymbol{X}\left(\boldsymbol{X}^{\prime} \boldsymbol{X}\right)^{-1} \boldsymbol{X}^{\prime}\left\{\boldsymbol{y}-\bar{y} \mathbf{1}_{n}\right\}\right\|^{2}}{\left\|\boldsymbol{y}-\bar{y} \mathbf{1}_{n}\right\|^{2}} .
$$

Maruyama and Strawderman (2006) proposed another class of improved generalized Bayes estimators. The proofs in all of these papers seem to depend strongly on the normality assumption. So it seems then, that it may be difficult or impossible to extend the dominance results to the non-normal case. Also many statisticians have thought that estimation of variance is more sensitive to the assumption of error distribution compared to estimation of the mean vector, where some robustness results have been derived by Maruyama and Strawderman (2005). 
Note that we use the term "robustness" in this sense of distributional robustness over the class of spherically symmetric error distributions. We specifically are not using the term to indicate a high breakdown point. The use of the term "robustness" in our sense is however common (if somewhat misleading) in the context of insensitivity to the error distribution in the context of shrinkage literature.

In this paper, we derive a class of generalized Bayes estimators relative to a class of separable priors of the form $\pi(\alpha, \boldsymbol{\beta})\left\{\sigma^{2}\right\}^{-1}$ and show that the resulting generalized Bayes estimator is independent of the form of the (spherically symmetric) sampling distribution. Additionally, we show, for a particular subclass of these separable priors, $\left(\boldsymbol{\beta}^{\prime} \boldsymbol{X}^{\prime} \boldsymbol{X} \boldsymbol{\beta}\right)^{-(p-2) / 2}\left\{\sigma^{2}\right\}^{-1}$, that the resulting robust generalized Bayes estimator has the additional robustness property of being minimax and dominating the unbiased estimator $\delta_{U}$ simultaneously, for the entire class of scale mixture of Gaussians.

A similar (but somewhat stronger) robustness property has been studied in the context of estimation of the vector of regression parameters $(\alpha, \boldsymbol{\beta})$ by Maruyama and Strawderman (2005). They gave separable priors of a form similar to priors in this paper for which the generalized Bayes estimators are minimax for the entire class of spherically symmetric distributions (and not just scale mixture of normals). We suspect that the distributional robustness property of the present paper also extends well beyond the class of scale mixture of normal distributions but have not been able to demonstrate just how much further it does extend.

The organization of this paper is as follows. In Section 2 we derive generalized Bayes estimators under separable priors and demonstrate that the resulting estimator is independent of the (spherically symmetric) sampling density. In Section 3 we show that a certain subclass of estimators which are minimax under normality remains minimax for the entire class of scale mixture of normals. Further, we show that certain generalized Bayes estimators studied in Section 2 have this (double) robustness property. Some comments are given in Section 4 and an appendix gives proofs of certain of the results.

\section{A generalized Bayes estimator with respect to the harmonic prior}

In this section, we show that the generalized Bayes estimator of the variance with respect to a certain class of priors is independent of the particular sampling model under Stein's loss. Also we will give an exact form of this estimator for a particular subclass of "(super)harmonic" priors that, we will later show, is minimax for a large subclass of spherically symmetric error distributions.

Theorem 2.1. The generalized Bayes estimator with respect to $\pi\left(\alpha, \boldsymbol{\beta}, \sigma^{2}\right)=$ $\pi(\alpha, \boldsymbol{\beta})\left\{\sigma^{2}\right\}^{-1}$ under Stein's loss (1.3) is independent of the particular spherically symmetric sampling model and hence is given by the generalized Bayes estimator under the Gaussian distribution.

Proof. See Appendix. 
Now let $p \geq 3$ and $\pi(\alpha, \boldsymbol{\beta})=\left(\boldsymbol{\beta}^{\prime} \boldsymbol{X}^{\prime} \boldsymbol{X} \boldsymbol{\beta}\right)^{-(p-a) / 2}$. This is related to a family of (super)harmonic functions as follows. If, in the above joint prior for $(\alpha, \boldsymbol{\beta})$, we make the change of variables, $\boldsymbol{\theta}=\left(\boldsymbol{X}^{\prime} \boldsymbol{X}\right)^{1 / 2} \boldsymbol{\beta}$, the joint prior of $(\alpha, \boldsymbol{\theta})$ becomes

$$
\pi(\alpha, \boldsymbol{\theta})=\|\boldsymbol{\theta}\|^{-(p-a)} .
$$

The Laplacian of $\|\boldsymbol{\theta}\|^{-(p-a)}$ is given by

$$
\sum_{i=1}^{p} \frac{\partial^{2}}{\partial \theta_{i}^{2}}\|\boldsymbol{\theta}\|^{-(p-a)}=(p-a)(2-a)\|\boldsymbol{\theta}\|^{-(p-a)-2},
$$

which is negative (i.e. super-harmonic) for $2<a<p$ and is zero (i.e. harmonic) for $a=2$.

Theorem 2.2. Under the model (1.1) with spherically symmetric error distribution (1.2) and Stein's loss (1.3), the generalized Bayes estimator with respect to $\pi\left(\alpha, \boldsymbol{\beta}, \sigma^{2}\right)=\left(\boldsymbol{\beta}^{\prime} \boldsymbol{X}^{\prime} \boldsymbol{X} \boldsymbol{\beta}\right)^{-(p-a) / 2}\left\{\sigma^{2}\right\}^{-1}$ for $0<a<p$ is given by

$$
\delta_{a}^{G B}=\phi_{a}^{G B}\left(R^{2}\right) \frac{\mathrm{RSS}}{n-p-1}
$$

where

$$
\phi_{a}^{G B}\left(R^{2}\right)=\frac{n-p-1}{n-a-1} \frac{\int_{0}^{1} t^{p / 2-a / 2-1}(1-t)^{a / 2-1}\left(1-R^{2} t\right)^{(n-p-a-1) / 2} d t}{\int_{0}^{1} t^{p / 2-a / 2-1}(1-t)^{a / 2-1}\left(1-R^{2} t\right)^{(n-p-a+1) / 2} d t} .
$$

Proof. See Appendix.

\section{Minimaxity}

In this section, we demonstrate robustness of minimaxity under scale mixture of normals for a class of estimators which are minimax under normality.

Theorem 3.1. Assume $\delta_{\phi}=\phi\left(R^{2}\right)\{\mathrm{RSS} /(n-p-1)\}$ where $\phi(\cdot)$ is monotone nondecreasing, improves on the unbiased estimator, $\delta_{U}$, under normality and Stein's loss. Then $\delta_{\phi}$ also improves on the unbiased estimator, $\delta_{U}$, under scale mixture of normals and Stein's loss.

Proof. Let $f$ be a scale mixture of normals where the scalar $\tau$ satisfies $E\left[\tau^{2}\right]=1$, that is,

$$
f(t)=\int_{0}^{\infty}(2 \pi \tau)^{-n / 2} \exp \left(-t /\left\{2 \tau^{2}\right\}\right) g\left(\tau^{2}\right) d \tau^{2} .
$$

Then $\boldsymbol{y} \mid \tau^{2} \sim N_{n}\left(\alpha \mathbf{1}_{n}+\boldsymbol{X} \boldsymbol{\beta}, \sigma^{2} \tau^{2} \boldsymbol{I}_{n}\right)$ and the risk difference between these 
estimators is given by

$$
\begin{aligned}
& R\left(\left\{\alpha, \boldsymbol{\beta}, \sigma^{2}\right\}, \delta_{U}\right)-R\left(\left\{\alpha, \boldsymbol{\beta}, \sigma^{2}\right\}, \delta_{\phi}\right) \\
& =E_{\boldsymbol{y}}\left[\frac{\left\{1-\phi\left(R^{2}\right)\right\}}{n-p-1} \frac{\mathrm{RSS}}{\sigma^{2}}+\log \phi\left(R^{2}\right)\right] \\
& =E_{\tau^{2}}\left[E_{\boldsymbol{y} \mid \tau^{2}}\left[\frac{\left\{1-\phi\left(R^{2}\right)\right\}}{n-p-1} \frac{\mathrm{RSS}}{\sigma^{2}}+\log \phi\left(R^{2}\right)\right]\right] \\
& =E_{\tau^{2}}\left[E_{\boldsymbol{y} \mid \tau^{2}}\left[\frac{\left\{1-\phi\left(R^{2}\right)\right\} \operatorname{RSS}}{(n-p-1)\left\{\sigma^{2} \tau^{2}\right\}}+\log \phi\left(R^{2}\right)\right]\right] \\
& +\frac{1}{n-p-1} E_{\tau^{2}}\left[E_{\boldsymbol{y} \mid \tau^{2}}\left[\frac{\left\{1-\phi\left(R^{2}\right)\right\} \operatorname{RSS}}{\sigma^{2} \tau^{2}}\left(\tau^{2}-1\right)\right]\right] .
\end{aligned}
$$

In the the first term of the right-hand side of the above equality,

$$
E_{\boldsymbol{y} \mid \tau^{2}}\left[\frac{\left\{1-\phi\left(R^{2}\right)\right\} \operatorname{RSS}}{(n-p-1)\left\{\sigma^{2} \tau^{2}\right\}}+\log \phi\left(R^{2}\right)\right]
$$

is the risk difference under the Gaussian assumption, which is given by

$$
R\left(\left\{\alpha, \boldsymbol{\beta}, \tau^{2} \sigma^{2}\right\}, \delta_{U}\right)-R\left(\left\{\alpha, \boldsymbol{\beta}, \tau^{2} \sigma^{2}\right\}, \delta_{\phi}\right)
$$

where $\boldsymbol{y} \sim N_{n}\left(\alpha \mathbf{1}_{n}+\boldsymbol{X} \boldsymbol{\beta}, \sigma^{2} \tau^{2} \boldsymbol{I}_{n}\right)$. From the assumption of the theorem, it is non-negative for any $\tau^{2}>0$. Hence it suffices to show that the second term is non-negative.

For given $\tau^{2},\left\{\left\|\boldsymbol{y}-\bar{y} \mathbf{1}_{n}\right\|^{2}-\operatorname{RSS}\right\} /\left\{\sigma^{2} \tau^{2}\right\}(=U)$ and $\operatorname{RSS} /\left\{\sigma^{2} \tau^{2}\right\}(=V)$ are independently distributed as $\chi_{p}^{2}\left(\lambda / \tau^{2}\right)$ with $\lambda=\boldsymbol{\beta}^{\prime} \boldsymbol{X}^{\prime} \boldsymbol{X} \boldsymbol{\beta} / \sigma^{2}$ and $\chi_{n-p-1}^{2}$. Since $R^{2}$ is given by $1-\operatorname{RSS} /\left\|\boldsymbol{y}-\bar{y} \mathbf{1}_{n}\right\|^{2}=(1+V / U)^{-1}$, the second term of the right-hand side of $(3.1)$ is written as

$$
\begin{aligned}
& E_{\tau^{2}}\left[E_{\boldsymbol{y} \mid \tau^{2}}\left[\frac{\left\{1-\phi\left(R^{2}\right)\right\} \operatorname{RSS}}{\sigma^{2} \tau^{2}}\left(\tau^{2}-1\right)\right]\right] \\
& =E_{\left\{U, V, \tau^{2}\right\}}\left[\left\{1-\phi\left(\{1+V / U\}^{-1}\right)\right\}\left(\tau^{2}-1\right) V\right] \\
& =E_{V}\left[E_{\tau^{2} \mid V}\left[\psi\left(\tau^{2}, V\right)\left(\tau^{2}-1\right)\right] V\right],
\end{aligned}
$$

where

$$
\psi\left(\tau^{2}, v\right)=1-E\left[\phi\left(\left\{1+v / \chi_{p}^{2}\left(\lambda / \tau^{2}\right)\right\}^{-1}\right)\right] .
$$

By the monotone likelihood ratio property of non-central $\chi^{2}, \psi\left(\tau^{2}, v\right)$ is nondecreasing in $\tau^{2}$ for any fixed $v$. Further, by the covariance inequality,

$$
E_{\tau^{2} \mid V}\left[\psi\left(\tau^{2}, V\right)\left(\tau^{2}-1\right)\right] \geq E_{\tau^{2} \mid V}\left[\tau^{2}-1\right] E_{\tau^{2} \mid V}\left[\psi\left(\tau^{2}, V\right)\right]=0
$$

since $V$ and $\tau^{2}$ are mutually independent and $E\left[\tau^{2}\right]=1$. The inequality (3.3) implies that the second term of the right-hand side of (3.1) is non-negative. 
Under the normality assumption, Brewster and Zidek (1974) showed that the estimator $\phi\left(R^{2}\right) \delta_{U}$ with nondecreasing $\phi$ dominates the unbiased estimator $\delta_{U}$ if $\phi^{B Z} \leq \phi \leq 1$, where $\phi^{B Z}$ is given by (1.6). Maruyama and Strawderman (2006) demonstrated that the generalized Bayes estimator of Theorem 2.2 with $a=2$ satisfies this condition. Hence our main result shows that the generalized Bayes estimator of Theorem 2.2 with $a=2$, is minimax for the entire class of variance mixture of normal distributions.

Theorem 3.2. Let $n-1>p \geq 3$. Under Stein's loss, the estimator given by

$$
\delta^{H}=\phi^{H}\left(R^{2}\right) \frac{\mathrm{RSS}}{n-p-1}
$$

where

$$
\phi^{H}\left(R^{2}\right)=\frac{n-p-1}{n-3} \frac{\int_{0}^{1} t^{p / 2-2}\left(1-R^{2} t\right)^{(n-p-3) / 2} d t}{\int_{0}^{1} t^{p / 2-2}\left(1-R^{2} t\right)^{(n-p-1) / 2} d t}
$$

is minimax and generalized Bayes with respect to the harmonic prior

$$
\pi\left(\alpha, \boldsymbol{\beta}, \sigma^{2}\right)=\left(\boldsymbol{\beta}^{\prime} \boldsymbol{X}^{\prime} \boldsymbol{X} \boldsymbol{\beta}\right)^{-(p-2) / 2}\left\{\sigma^{2}\right\}^{-1}
$$

for the entire class of scale mixture of normals.

Remark 3.1. Note that the coefficient of determination is given in (1.7) and that the expectations of the numerator and the denominator are given by

$$
\begin{aligned}
& E\left[\left\|\boldsymbol{X}\left(\boldsymbol{X}^{\prime} \boldsymbol{X}\right)^{-1} \boldsymbol{X}^{\prime}\left\{\boldsymbol{y}-\bar{y} \mathbf{1}_{n}\right\}\right\|^{2}\right]=\sigma^{2}\{\xi+p\}, \\
& E\left[\left\|\boldsymbol{y}-\bar{y} \mathbf{1}_{n}\right\|^{2}\right]=\sigma^{2}\{\xi+n-1\},
\end{aligned}
$$

where $\xi=\boldsymbol{\beta}^{\prime} \boldsymbol{X}^{\prime} \boldsymbol{X} \boldsymbol{\beta} / \sigma^{2}$. Hence the smaller $R^{2}$ corresponds to the smaller $\xi$ since $n-1>p$.

Our class of improved estimators utilizes the coefficient of determination $R^{2}$ in making a (smooth) choice between $\delta_{U}$ (when $R^{2}$ and $\xi$ are large) and $\| \boldsymbol{y}-$ $\bar{y} \mathbf{1}_{n} \|^{2} /(n-1)$ (when $R^{2}$ and $\xi$ are small) and reflects the relatively common knowledge among statisticians, that $\left\|\boldsymbol{y}-\bar{y} \mathbf{1}_{n}\right\|^{2} /(n-1)$ is stochastically closer to $\sigma^{2}$ when $R^{2}$ is small.

Remark 3.2. The estimator $\delta^{H}$ is not the only minimax generalized Bayes estimator under scale mixture of normals. In Theorem 2.2, we also provided the generalized Bayes estimator with respect to superharmonic prior given by $\pi\left(\alpha, \boldsymbol{\beta}, \sigma^{2}\right)=\left(\boldsymbol{\beta}^{\prime} \boldsymbol{X}^{\prime} \boldsymbol{X} \boldsymbol{\beta}\right)^{-(p-a) / 2}\left\{\sigma^{2}\right\}^{-1}$. In Maruyama and Strawderman (2006), we show that for $\delta_{a}^{G B}$ with $2<a(n, p) \leq a<p$ is minimax in the normal case with a monotone $\phi_{a}^{G B}$. Hence for $a$ in this range $\delta_{a}^{G B}$ is also minimax and generalized Bayes for the entire class of scale mixture of normals. The bound $a(n, p)$ has a somewhat complicated form and we omit the details (however, see Maruyama and Strawderman (2006) for details).

Note that $\delta^{H}$ corresponds to $\delta_{a}^{G B}$ with $a=2$ since the corresponding prior to $\delta^{H}$ is given by (3.6). Note also that the unbiased estimator $\delta_{U}$ which is derived as the Jeffrey's prior $\pi\left(\alpha, \boldsymbol{\beta}, \sigma^{2}\right)=1 / \sigma^{2}$ corresponds to $\lim _{a \rightarrow p} \delta_{a}^{G B}$. Therefore we conjecture that $\delta_{a}^{G B}$ with any $a \in(2, p)$ is minimax. 
Remark 3.3. Under the normality assumption, Maruyama and Strawderman (2006) gave a subclass of minimax generalized Bayes estimators with the particularly simple form

$$
\delta^{S B}=\left\{1+c\left(1-R^{2}\right)\right\}^{-1} \frac{\mathrm{RSS}}{n-p-1}
$$

for $0<c \leq c(n, p)$ where $c(n, p)$ has a slightly complicated form, which we omit (see Maruyama and Strawderman (2006) for details). Under spherical symmetry, this estimator is not necessarily derived as generalized Bayes (See the following Remark), but is still minimax under scale mixture of normals.

Remark 3.4. Interestingly, when $(n-1) / 2<p<(n-1)$, the generalized Bayes estimator with respect to $\left\|\boldsymbol{\beta}^{\prime} \boldsymbol{X}^{\prime} \boldsymbol{X} \boldsymbol{\beta}\right\|^{-p+(n-1) / 2}\left\{\sigma^{2}\right\}^{-1}$ is given by

$$
\delta_{*}^{S B}=\left(1+\frac{2 p-n+1}{n-p-1}\left(1-R^{2}\right)\right)^{-1} \frac{\mathrm{RSS}}{n-p-1}
$$

for the entire class of spherically symmetric distributions (See Maruyama and Strawderman (2006) for the technical details). Hence when

$$
(2 p-n+1) /(n-p-1) \leq c(n, p)
$$

$\delta_{*}^{S B}$ is minimax and generalized Bayes for the entire class of scale mixture of normals. Unfortunately, numerical calculations indicate that, for $n$ in the range $(25,10,000)$, the inequality $(3.9)$ is only satisfied for $p=(n+1) / 2$ for $n$ odd and $n / 2$ and $n / 2+1$ for $n$ even.

Actually, under the Gaussian assumption, $\delta^{S B}$ given in (3.7) with $c$ larger than $c(n, p)$ can be demonstrated to be minimax numerically even though our analytic upper bound on $c$ for minimaxity is $c(n, p)$. In practice, since $\phi^{H}$ given in (3.5) can be calculated quickly and precisely, we recommend the use of $\delta^{H}$ given in (3.4).

Remark 3.5. For Theorems 2.1, 3.1 and 3.2, the choice of the loss function is the key. Many of the results introduced in Section 1 were initially proved under the quadratic loss function $\left(\delta / \sigma^{2}-1\right)^{2}$. Under the Gaussian assumption, the corresponding results can be obtained by replacing $n+2$ by $n$. On the other hand, the generalized Bayes estimator with respect to $\pi\left(\alpha, \boldsymbol{\beta}, \sigma^{2}\right)=\pi(\alpha, \boldsymbol{\beta})\left\{\sigma^{2}\right\}^{-1}$ depends on the particular sampling model and hence robustness results do not hold under non-Gaussian assumption.

\section{Concluding Remarks}

In this paper, we have studied estimation of the error variance in a general linear model with a spherically symmetric error distribution. We have shown, under Stein's loss, that separable priors of the form $\pi(\alpha, \boldsymbol{\beta})\left\{\sigma^{2}\right\}^{-1}$ have associated generalized Bayes estimators which are independent of the form of the (spherically symmetric) sampling distribution. We have further exhibited a subclass of 
"superharmonic" priors for which these generalized Bayes estimators dominate the usual unbiased and best equivariant estimator, $\delta_{U}$, for the entire class of scale mixture of normal error distributions.

We have previously studied a very similar class of prior distributions in the problem of estimating the regression coefficients $(\alpha, \boldsymbol{\beta})$ under quadratic loss (See Maruyama and Strawderman (2005)). In that study we demonstrated a similar double robustness property: to wit, that the generalized Bayes estimators are independent of the form of the sampling distribution and that they are minimax over the entire class of spherically symmetric distributions.

The main difference between the classes of priors in the two settings are a) in the present study, the prior on $\sigma^{2}$ is proportional to $\left\{\sigma^{2}\right\}^{-1}$ while it is proportional to $\left\{\sigma^{2}\right\}^{a}$ in the earlier study; and b) in this paper, the prior on $(\alpha, \boldsymbol{\beta})$ is also separable with $\alpha$ being uniform on the real line and $\boldsymbol{\beta}$ having the "superharmonic" form, while in the earlier paper $(\alpha, \boldsymbol{\beta})$ jointly had the superharmonic form.

The difference a) is essential since a prior on $\sigma^{2}$ proportional to $\left\{\sigma^{2}\right\}^{-1}$ gives the best equivariant and minimax estimator $\delta_{U}$, while such a restriction is not necessary when estimating the regression parameters $(\alpha, \boldsymbol{\beta})$.

The difference in b) is inessential, and either form of priors on the regression parameters $(\alpha, \boldsymbol{\beta})$ will give estimators with the double robustness properties in each of the problems studied. The form of the estimators, of course, will be somewhat different. In the case of the present paper, the main difference would be to replace $n-p-1$ by $n-p$ and to replace $R^{2}$ by

$$
\left\{n \bar{y}^{2}+\left\|\boldsymbol{X}\left(\boldsymbol{X}^{\prime} \boldsymbol{X}\right)^{-1} \boldsymbol{X}^{\prime} \boldsymbol{y}\right\|^{2}\right\} /\|\boldsymbol{y}\|^{2} .
$$

As a consequence, the results in these papers suggest that separable priors, and in particular the "harmonic" prior given (3.6), are very worthy candidates as objective priors in regression problems. They produce generalized Bayes minimax procedures dominating the classical unbiased, best equivariant estimators of both regression parameters and scale parameters simultaneously and uniformly over a broad class of spherically symmetric error distributions.

\section{Appendix A: Proof of Theorem 2.1}

The (generalized) Bayes estimator with Stein's loss is given by $\left\{E\left[1 / \sigma^{2} \mid \boldsymbol{y}\right]\right\}^{-1}$. Under the improper density $\pi\left(\alpha, \boldsymbol{\beta}, \sigma^{2}\right)=\pi(\alpha, \boldsymbol{\beta})\left\{\sigma^{2}\right\}^{-1}$, the generalized Bayes estimator is given by

$$
\frac{\iint m_{0}^{f}(\boldsymbol{y} \mid \alpha, \boldsymbol{\beta}) \pi(\alpha, \boldsymbol{\beta}) d \alpha d \boldsymbol{\beta}}{\iint m_{1}^{f}(\boldsymbol{y} \mid \alpha, \boldsymbol{\beta}) \pi(\alpha, \boldsymbol{\beta}) d \alpha d \boldsymbol{\beta}}
$$

where $m_{i}^{f}(\boldsymbol{y} \mid \alpha, \boldsymbol{\beta})$ for $i=0,1$ is the conditional marginal density of $\boldsymbol{y}$ with respect to $\left\{\sigma^{2}\right\}^{-1-i}$ given $\alpha$ and $\boldsymbol{\beta}$,

$$
m_{i}^{f}(\boldsymbol{y} \mid \alpha, \boldsymbol{\beta})=\int_{0}^{\infty} \sigma^{-n} f\left(\frac{\left\|\boldsymbol{y}-\alpha \mathbf{1}_{n}-\boldsymbol{X} \boldsymbol{\beta}\right\|^{2}}{\sigma^{2}}\right)\left(\sigma^{2}\right)^{-i-1} d \sigma^{2} .
$$


Further we have

$$
\begin{aligned}
& m_{i}^{f}(\boldsymbol{y} \mid \alpha, \boldsymbol{\beta})=\left\|\boldsymbol{y}-\alpha \mathbf{1}_{n}-\boldsymbol{X} \boldsymbol{\beta}\right\|^{-n-2 i} \int_{0}^{\infty} t^{\{n+2 i\} / 2-1} f(t) d t \\
& =\frac{\int_{0}^{\infty} t^{(n+2 i) / 2-1} f(t) d t}{\int_{0}^{\infty} t^{(n+2 i) / 2-1} f_{G}(t) d t} \int_{0}^{\infty} f_{G}\left(\frac{\left\|\boldsymbol{y}-\alpha \mathbf{1}_{n}-\boldsymbol{X} \boldsymbol{\beta}\right\|^{2}}{\sigma^{2}}\right) \frac{\left(\sigma^{2}\right)^{-i-1}}{\sigma^{n}} d \sigma^{2}
\end{aligned}
$$

where

$$
f_{G}(t)=\frac{1}{(2 \pi)^{n / 2}} \exp (-t / 2)
$$

Hence the generalized Bayes estimator is

$$
\frac{\iint m_{0}^{f}(\boldsymbol{y} \mid \alpha, \boldsymbol{\beta}) \pi(\alpha, \boldsymbol{\beta}) d \alpha d \boldsymbol{\beta}}{\iint m_{1}^{f}(\boldsymbol{y} \mid \alpha, \boldsymbol{\beta}) \pi(\alpha, \boldsymbol{\beta}) d \alpha d \boldsymbol{\beta}}=\frac{\int_{0}^{\infty} t^{n / 2-1} f(t) d t}{\int_{0}^{\infty} t^{n / 2} f(t) d t} \frac{\int_{0}^{\infty} t^{n / 2} f_{G}(t) d t}{\int_{0}^{\infty} t^{n / 2-1} f_{G}(t) d t} \frac{m_{0}^{G}(\boldsymbol{y})}{m_{1}^{G}(\boldsymbol{y})}
$$

where

$$
m_{i}^{G}(\boldsymbol{y})=\iiint f_{G}\left(\frac{\left\|\boldsymbol{y}-\alpha \mathbf{1}_{n}-\boldsymbol{X} \boldsymbol{\beta}\right\|^{2}}{\sigma^{2}}\right) \frac{\pi(\alpha, \boldsymbol{\beta})}{\left(\sigma^{2}\right)^{n / 2+i+1}} d \alpha d \boldsymbol{\beta} d \sigma^{2} .
$$

Since $\boldsymbol{\epsilon}$ has a spherically symmetric density $f\left(\boldsymbol{\epsilon}^{\prime} \boldsymbol{\epsilon}\right)$ and $E[\boldsymbol{\epsilon}]=\mathbf{0}_{n}$ and $\operatorname{Var}[\boldsymbol{\epsilon}]=$ $\boldsymbol{I}_{n}, f$ as well as $f_{G}$ satisfies

$$
\int_{\mathcal{R}^{n}} f\left(\boldsymbol{\epsilon}^{\prime} \boldsymbol{\epsilon}\right) d \epsilon=\frac{\pi^{n / 2}}{\Gamma(n / 2)} \int_{0}^{\infty} s^{n / 2-1} f(s) d s=1,
$$

and

$$
\int_{\mathcal{R}^{n}} \boldsymbol{\epsilon}^{\prime} \boldsymbol{\epsilon} f\left(\boldsymbol{\epsilon}^{\prime} \boldsymbol{\epsilon}\right) d \epsilon=\frac{\pi^{n / 2}}{\Gamma(n / 2)} \int_{0}^{\infty} s^{n / 2} f(s) d s=n .
$$

Hence we have

$$
\frac{\int_{0}^{\infty} t^{n / 2-1} f(t) d t}{\int_{0}^{\infty} t^{n / 2} f(t) d t} \frac{\int_{0}^{\infty} t^{n / 2} f_{G}(t) d t}{\int_{0}^{\infty} t^{n / 2-1} f_{G}(t) d t}=\frac{1}{n} \cdot \frac{n}{1}=1
$$

and hence the generalized Bayes estimator is given by $m_{0}^{G}(\boldsymbol{y}) / m_{1}^{G}(\boldsymbol{y})$ which is independent of $f$.

\section{Appendix B: Proof of Theorem 2.2}

Note, for $0<a<p$,

$$
\begin{aligned}
\left(\boldsymbol{\beta}^{\prime} \boldsymbol{X}^{\prime} \boldsymbol{X} \boldsymbol{\beta}\right)^{-(p-a) / 2} & =\frac{2^{a / 2} \pi^{p / 2}}{\Gamma(\{p-a\} / 2)\left|\boldsymbol{X}^{\prime} \boldsymbol{X}\right|^{1 / 2}} \\
& \times\left\{\sigma^{2}\right\}^{a / 2} \int_{0}^{\infty} g^{a / 2-1} \frac{\left|\boldsymbol{X}^{\prime} \boldsymbol{X}\right|^{1 / 2}}{\left(2 \pi \sigma^{2} g\right)^{p / 2}} \exp \left(-\frac{\boldsymbol{\beta}^{\prime} \boldsymbol{X}^{\prime} \boldsymbol{X} \boldsymbol{\beta}}{2 \sigma^{2} g}\right) d g .
\end{aligned}
$$


Then

$$
\begin{aligned}
m_{i}^{G}(\boldsymbol{y}) & =A \int_{-\infty}^{\infty} \int_{R^{p}} \int_{0}^{\infty} \int_{0}^{\infty} \frac{1}{\left(2 \pi \sigma^{2}\right)^{n / 2}} \exp \left(-\frac{\left\|\boldsymbol{y}-\alpha \mathbf{1}_{n}-\boldsymbol{X} \boldsymbol{\beta}\right\|^{2}}{2 \sigma^{2}}\right) \\
\times & \frac{g^{a / 2-1}}{\left\{\sigma^{2}\right\}^{-a / 2+1+i}} \frac{\left|\boldsymbol{X}^{\prime} \boldsymbol{X}\right|^{1 / 2}}{\left(2 \pi \sigma^{2}\right)^{p / 2} g^{p / 2}} \exp \left(-\frac{\boldsymbol{\beta}^{\prime} \boldsymbol{X}^{\prime} \boldsymbol{X} \boldsymbol{\beta}}{2 \sigma^{2} g}\right) d \alpha d \boldsymbol{\beta} d \sigma^{2} d g,
\end{aligned}
$$

where $A=\left\{2^{a / 2} \pi^{p / 2}\right\} /\left\{\Gamma(\{p-a\} / 2)\left|\boldsymbol{X}^{\prime} \boldsymbol{X}\right|^{1 / 2}\right\}$. In the following, we calculate the integration in (B.2) with respect to $\alpha, \boldsymbol{\beta}, \sigma^{2}$, and $g$, in this order.

By the simple relation

$$
\boldsymbol{y}-\alpha \mathbf{1}_{n}-\boldsymbol{X} \boldsymbol{\beta}=(-\alpha+\bar{y}) \mathbf{1}_{n}+\boldsymbol{v}-\boldsymbol{X} \boldsymbol{\beta}
$$

where $\bar{y}$ mean the mean of $\boldsymbol{y}$ and $\boldsymbol{v}=\boldsymbol{y}-\bar{y} \mathbf{1}_{n}$, we have the Pythagorean relation,

$$
\left\|\boldsymbol{y}-\alpha \mathbf{1}_{n}-\boldsymbol{X} \boldsymbol{\beta}\right\|^{2}=n(-\alpha+\bar{y})^{2}+\|\boldsymbol{v}-\boldsymbol{X} \boldsymbol{\beta}\|^{2},
$$

since $\boldsymbol{X}$ has been already centered. Then we have

$$
\begin{aligned}
& \int_{-\infty}^{\infty} \frac{1}{\left(2 \pi \sigma^{2}\right)^{n / 2}} \exp \left(-\frac{\left\|\boldsymbol{y}-\alpha \mathbf{1}_{n}-\boldsymbol{X} \boldsymbol{\beta}\right\|^{2}}{2 \sigma^{2}}\right) d \alpha \\
& =\frac{n^{1 / 2}}{\left(2 \pi \sigma^{2}\right)^{(n-1) / 2}} \exp \left(-\frac{\|\boldsymbol{v}-\boldsymbol{X} \boldsymbol{\beta}\|^{2}}{2 \sigma^{2}}\right) .
\end{aligned}
$$

Next we consider the integration with respect to $\boldsymbol{\beta}$. Note the relation of completing squares with respect to $\boldsymbol{\beta}$

$$
\begin{aligned}
& \|\boldsymbol{v}-\boldsymbol{X} \boldsymbol{\beta}\|^{2}+g^{-1} \boldsymbol{\beta}^{\prime} \boldsymbol{X}^{\prime} \boldsymbol{X} \boldsymbol{\beta} \\
& =\frac{1+g}{g}\left(\boldsymbol{\beta}-\frac{g}{1+g} \hat{\boldsymbol{\beta}}\right)^{\prime} \boldsymbol{X}^{\prime} \boldsymbol{X}\left(\boldsymbol{\beta}-\frac{g}{1+g} \hat{\boldsymbol{\beta}}\right)+\frac{\|\boldsymbol{v}\|^{2}}{1+g}\left\{g\left(1-R^{2}\right)+1\right\}
\end{aligned}
$$

where $\hat{\boldsymbol{\beta}}=\left(\boldsymbol{X}^{\prime} \boldsymbol{X}\right)^{-1} \boldsymbol{X}^{\prime} \boldsymbol{v}$ and $R^{2}=\|\boldsymbol{X} \hat{\boldsymbol{\beta}}\|^{2} /\|\boldsymbol{v}\|^{2}$ is the coefficient of determination. Hence we have

$$
\begin{aligned}
& \int_{-\infty}^{\infty} \int_{R^{p}} \frac{1}{\left(2 \pi \sigma^{2}\right)^{n / 2}} \exp \left(-\frac{\left\|\boldsymbol{y}-\alpha \mathbf{1}_{n}-\boldsymbol{X} \boldsymbol{\beta}\right\|^{2}}{2 \sigma^{2}}\right) \\
& \times \frac{\left|\boldsymbol{X}^{\prime} \boldsymbol{X}\right|^{1 / 2}}{\left(2 \pi \sigma^{2}\right)^{p / 2} g^{p / 2}} \exp \left(-\frac{\boldsymbol{\beta}^{\prime} \boldsymbol{X}^{\prime} \boldsymbol{X} \boldsymbol{\beta}}{2 \sigma^{2} g}\right) d \alpha d \boldsymbol{\beta} \\
& \quad=\frac{n^{1 / 2}(1+g)^{-p / 2}}{\left(2 \pi \sigma^{2}\right)^{(n-1) / 2}} \exp \left(-\frac{\|\boldsymbol{v}\|^{2}\left\{g\left(1-R^{2}\right)+1\right\}}{2 \sigma^{2}(g+1)}\right) .
\end{aligned}
$$

Next we consider integration with respect to $\sigma^{2}$. By (B.3), we have

$$
\begin{aligned}
& \int_{-\infty}^{\infty} \int_{R^{p}} \int_{0}^{\infty} \frac{1}{\left(2 \pi \sigma^{2}\right)^{n / 2}} \exp \left(-\frac{\left\|\boldsymbol{y}-\alpha \mathbf{1}_{n}-\boldsymbol{X} \boldsymbol{\beta}\right\|^{2}}{2 \sigma^{2}}\right) \\
& \quad \times \frac{\left|\boldsymbol{X}^{\prime} \boldsymbol{X}\right|^{1 / 2}}{\left(2 \pi \sigma^{2}\right)^{p / 2} g^{p / 2}} \exp \left(-\frac{\boldsymbol{\beta}^{\prime} \boldsymbol{X}^{\prime} \boldsymbol{X} \boldsymbol{\beta}}{2 \sigma^{2} g}\right)\left\{\sigma^{2}\right\}^{a / 2-1-i} d \alpha d \boldsymbol{\beta} d \sigma^{2} \\
& \quad=\frac{2^{-a / 2+i} n^{1 / 2} \Gamma(\{n-a-1+2 i\} / 2)}{\pi^{(n-1) / 2}\|\boldsymbol{v}\|^{n-a-1+2 i}} \frac{(1+g)^{(n-p-a-1+2 i) / 2}}{\left\{g\left(1-R^{2}\right)+1\right\}^{(n-a-1+2 i) / 2}} .
\end{aligned}
$$


Finally we consider integration with respect to $g$. By (B.4) we have

$$
\begin{aligned}
m_{i}^{G}(\boldsymbol{y})= & A \frac{2^{-a / 2+i} n^{1 / 2} \Gamma(\{n-a-1+2 i\} / 2)}{\pi^{(n-1) / 2}\|\boldsymbol{v}\|^{n-a-1+2 i}} \\
& \times \int_{0}^{\infty} \frac{g^{a / 2-1}(1+g)^{(n-p-a-1+2 i) / 2}}{\left\{g\left(1-R^{2}\right)+1\right\}^{(n-a-1+2 i) / 2}} d g \\
= & A \frac{2^{-a / 2+i} n^{1 / 2} \Gamma(\{n-a-1+2 i\} / 2)}{\pi^{(n-1) / 2}\|\boldsymbol{v}\|^{n-a-1+2 i}\left(1-R^{2}\right)^{(n-p-1-2 i) / 2}} \\
& \times \int_{0}^{1} t^{p / 2-a / 2-1}(1-t)^{a / 2-1}\left(1-R^{2} t\right)^{(n-p-a-1+2 i) / 2} d t .
\end{aligned}
$$

The second equality follows from the change of variables $1 /\left\{1+g\left(1-R^{2}\right)\right\} \rightarrow t$. By using the relation $\left(1-R^{2}\right)\left\|\boldsymbol{y}-\bar{y} \mathbf{1}_{n}\right\|^{2}=\mathrm{RSS}, m_{0}^{G}(\boldsymbol{y}) / m_{1}^{G}(\boldsymbol{y})$ is written as (2.2).

\section{Acknowledgements}

We are very grateful to the associate editor, and referees for wonderful insights which substantially helped us to strengthen this paper.

\section{References}

Brewster, J. F. and ZideK, J. V. (1974). Improving on equivariant estimators. Ann. Statist. 2 21-38. MR0381098

James, W. and Stein, C. (1961). Estimation with quadratic loss. In Proc. 4th Berkeley Sympos. Math. Statist. and Prob., Vol. I 361-379. Univ. California Press, Berkeley, Calif. MR0133191

Maruyama, Y. and Strawderman, W. E. (2005). A new class of generalized Bayes minimax ridge regression estimators. Ann. Statist. 33 1753-1770. MR2166561

Maruyama, Y. and Strawderman, W. E. (2006). A new class of minimax generalized Bayes estimators of a normal variance. J. Statist. Plann. Inference 136 3822-3836. MR2299167

Stein, C. (1964). Inadmissibility of the usual estimator for the variance of a normal distribution with unknown mean. Ann. Inst. Statist. Math. 16 155160. MR0171344

StraWderman, W. E. (1974). Minimax estimation of powers of the variance of a normal population under squared error loss. Ann. Statist. 2 190-198. MR0343442 